\title{
Gesture production patterns in aphasic discourse: In-depth description and preliminary predictions
}

\author{
Kazuki Sekine, Miranda L. Rose, Abby M. Foster, Michelle C. Attard \& Lucette \\ E. Lanyon
}

To cite this article: Kazuki Sekine, Miranda L. Rose, Abby M. Foster, Michelle C. Attard \& Lucette E. Lanyon (2013) Gesture production patterns in aphasic discourse: Indepth description and preliminary predictions, Aphasiology, 27:9, 1031-1049, DOI: 10.1080/02687038.2013.803017

To link to this article: http://dx.doi.org/10.1080/02687038.2013.803017

曲 Published online: 11 Jun 2013.

Submit your article to this journal $๘$

Џ Article views: 455

Q View related articles $\square$

Citing articles: 6 View citing articles 5 


\title{
Gesture production patterns in aphasic discourse: In-depth description and preliminary predictions
}

\author{
Kazuki Sekine ${ }^{1,2}$, Miranda L. Rose ${ }^{3,4}$, Abby M. Foster ${ }^{3,4,5}$, \\ Michelle C. Attard ${ }^{3,4}$, and Lucette E. Lanyon ${ }^{3,4}$
}

\author{
${ }^{1}$ School of Psychology, University of Birmingham, Birmingham, UK \\ ${ }^{2}$ Japan Society for the Promotion of Science, Tokyo, Japan \\ ${ }^{3}$ Department of Human Communication Sciences, Faculty of Health Sciences, La \\ Trobe University, Melbourne, Australia \\ ${ }^{4}$ Centre for Clinical Research Excellence in Aphasia Rehabilitation, School of \\ Health and Rehabilitation Sciences, The University of Queensland, Brisbane, \\ Australia \\ ${ }^{5}$ School of Health and Rehabilitation Sciences, The University of Queensland, \\ Brisbane, Australia
}

Background: Gesture frequently accompanies speech in healthy speakers. For many individuals with aphasia, gestures are a target of speech-language pathology intervention, either as an alternative form of communication or as a facilitative device for language restoration. The patterns of gesture production for people with aphasia and the participant variables that predict these patterns remain unclear.

Aims: We aimed to examine gesture production during conversational discourse in a large sample of individuals with aphasia. We used a detailed gesture coding system to determine patterns of gesture production associated with specific aphasia types and severities. Methods \& Procedures: We analysed conversation samples from AphasiaBank, gathered from 46 people with post-stroke aphasia and 10 healthy matched controls all of whom had gestured at least once during a story re-tell task. Twelve gesture types were coded. Descriptive statistics were used to describe the patterns of gesture production. Possible significant differences in production patterns according to aphasia type and severity were examined with a series of analyses of variance (ANOVA) statistics, and multiple regression analysis was used to examine these potential predictors of gesture production patterns.

Outcomes \& Results: Individuals with aphasia gestured significantly more frequently than healthy controls. Aphasia type and severity impacted significantly on gesture type in specific identified patterns detailed here, especially on the production of meaning-laden gestures.

Conclusions: These patterns suggest the opportunity for gestures as targets of aphasia therapy. Aphasia fluency accounted for a greater degree of data variability than aphasia severity or naming skills. More work is required to delineate predictive factors.

Keywords: Gesture; Aphasia; Natural conversation; Fluency; Severity.

Address correspondence to: Kazuki Sekine, School of Psychology, University of Birmingham, Edgbaston, Birmingham, B15 2TT, UK. E-mail: kazuki@tkc.att.ne.jp 


\section{INTRODUCTION}

A considerable body of evidence supports the notion that arm and hand gestures accompany human speech in predictable ways. A tight gesture/speech temporal relationship has been demonstrated (Morrel-Samuels \& Krauss, 1992) and various types of gestures have been defined (see Figure 1). These gestures accompany different types of speech components and acts (McNeill, 2000). At times part of the proposition being conveyed is displayed in gesture, either in conjunction with speech (Kendon, 1980), or in place of speech (McNeill, 2005). Propositions heavy in spatial information have been shown to have higher levels of accompanying iconic gesture (Kita \& Ozyurek, 2003), and in noisy environments when speech is difficult to process, gesture begins to take on language-like, analytic, and synthetic properties (McNeil, 2000; Melinger \& Levelt, 2004). Restricting gesture in healthy speakers has been shown to lead to increased dysfluency for speech with spatial content (Frick \& Guttentag, 1998; Rauscher, Krauss, \& Chen, 1996), and to poorer word retrieval (Pyers et al., 2010), although there is some controversy on this latter point (Beattie \& Coughlan, 1998). Such strong relationships between gesture and speech production have underpinned two major theories concerning the functions of gesture: gesture as a communication device, and gesture as a lexical facilitation device (see de Ruiter, 2000, for a review of models and theories).

Aphasia, a language disorder resulting from stroke and other acquired brain impairments, is a highly prevalent communication disorder. Within the stroke survivor population alone, it is estimated that $30 \%$ develop aphasia immediately post-stroke (Dickey, 2010; Engelter, 2006). Aphasia has devastating impacts on social and psychological health (Hilari et al., 2010), disrupts everyday work and leisure activities (Daniloff, Fritelli, Buckingham, Hoffman, \& Daniloff, 1986), and impairs quality of life (Cruice, Worrall, Hickson, \& Murison, 2005). Finding effective treatments and communication methods for people with aphasia is an important healthcare and social service goal.

One potentially potent non-verbal communication device for individuals with aphasia is gesture (Rose, 2006). Further, there is a considerable history in the speech pathology rehabilitation literature concerning gesture as a therapeutic modality aimed at language restoration (see Rose, Raymer, Lanyon \& Attard, this issue, for a systematic review). However, there is considerable variability in the population of people with aphasia in terms of severity, type, and co-morbid cognitive impairments, and it is likely that their gesture abilities also vary. Understanding the patterns of gesture production for people with aphasia is an important goal for aphasia rehabilitation. This current study aimed to reveal the relationship between gesture type and aphasia type.

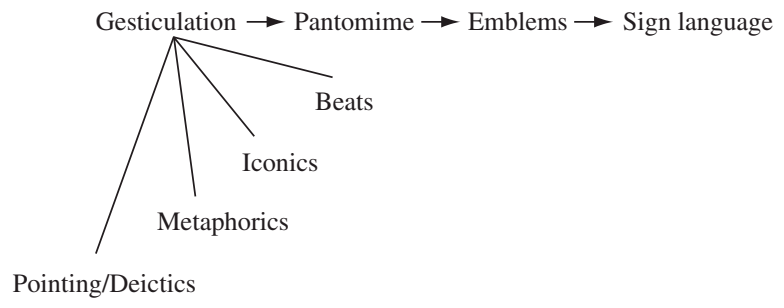

Figure 1. McNeill's continuum of gestures (adapted from McNeill, 2000). 
The findings from this study may provide guidance to clinicians considering the use of gestures in aphasia therapy.

To date, there has been very little high level evidence concerning the gesture production abilities of individuals with aphasia. The majority of studies have been small in nature, underpowered, and restricted in the range of aphasia types and severities investigated. Further, the range of gestures studied has been limited and discourse samples have often been extremely short. For example, Glosser, Wiener, and Kaplan (1986) studied five people with mild aphasia and five with moderate aphasia in two 5-minute conversations about their past experiences, with and without a visual restriction between interlocutors. They found a significant negative correlation between aphasia severity and gesture complexity. Similarly, Cicone Wapner, Foldi, Zurif, and Gardner (1979) studied the gestures of four people with aphasia, sampled from nine episodes of gesture produced during an interview-based conversation. Both Glosser et al. (1986) and Cicone et al. (1979) argued that people with aphasia are impaired in their gestural communication competence in parallel with their verbal communication competence. Several studies have also shown differences between people with fluent aphasia (e.g., Wernicke's) and non-fluent aphasia (e.g., Broca's) in referential tasks, such that people with Broca's aphasia are slow to initiate movement, have long pauses between movements (Duffy, Duffy, \& Mercaitis, 1984). Further, in discourse tasks people with Broca's aphasia have been shown to have frequent use of iconic gestures and beats (Behrmann \& Penn, 1984), and produce more referentially clear gestures (Cicone et al., 1979; Glosser et al., 1986).

However, in several other studies, individuals with aphasia were found to use gesture more frequently than healthy control speakers (Ahlsen, 1991; Le May, David, \& Thomas, 1988; Lott, 1999; Pedelty, 1987), and aphasia type interacted with the variety of gestures produced. In Lott's (1999) study of 15 individuals with aphasia (5 Broca's, 5 Wernicke's, and 5 anomic) and 15 healthy controls, individuals with Broca's aphasia produced the most iconic and meaning-laden gestures (e.g., iconics, emblems, and pantomimes) during story re-tell, and all three aphasic groups gestured more than their healthy peers. This was true both when measured in a ratio of gestures per 100 spoken words and in total overall numbers. Recently, Carlomagno, Pandolfi, Martini, Di Iasi, and Cristilli (2005) showed that 11 individuals with mildmoderate anomic aphasia produced four times more iconic gestures per spoken word than normal control speakers in a referential communication task.

Strong relationships have been documented between semantic processing abilities and gestural diversity, and limb apraxia and gesture comprehensibility in a story re-tell task for 24 individuals with severe aphasia (Hogrefe, Zeigler, Weidinger, \& Goldenberg, 2012). However, no relationship was found between aphasia severity and either gesture diversity or comprehensibility. Importantly, the participants represented a very restricted group of people with severe aphasia so that the lack of association between severity and gesture production may have been an artifact of the restricted sample. Further, it is not clear that these findings would generalise to the wider population of individuals with mild or moderate aphasia.

In a recent large-scale study, gesture production patterns were investigated in 98 individuals with aphasia and 64 healthy controls during the Cinderella story retell task (Sekine \& Rose, in press). A wide range of aphasia types and severities were represented in the sample. Sekine and Rose examined whether each of 12 specific types of gesticulations, emblems, and pantomimes were produced at least once by each individual. They found that significantly more individuals with aphasia gestured 
than the healthy controls. Further, individuals with aphasia produced four gesture types during story re-tell that were never produced by the healthy speakers: pointing to objects in space, pointing to self, pantomime, and letter gestures. While all individuals with aphasia used emblems and pointing to objects in space, a significantly higher proportion of individuals with Broca's aphasia produced iconic, pantomime, and number gestures as compared to individuals with other types of aphasia.

One limitation of the Sekine and Rose (in press) study was the screening level of assessment utilised. As noted, they examined whether individuals with aphasia were capable of producing each gesture type as least once during the story re-tells. While such screening information could be useful for clinicians wishing to make a quick assessment of potential gesture ability, a detailed analysis of gesture production frequency would provide a more comprehensive description of the gesture production patterns of individuals with aphasia. Further, analysis of conversational discourse might produce a more ecologically valid result than analysis of story re-tells.

\section{AIMS AND HYPOTHESES}

The aims of the current study were to: (1) investigate the gesture production patterns of individuals from a wide range of aphasia types and severities during conversation and compare this to data from healthy controls; (2) explore the impact of speech fluency, aphasia severity, and naming ability on the production of iconic gesture forms. These findings may contribute to refining communication therapies that harness iconic gestures.

Specifically, we hypothesised that

(1) Individuals with aphasia will produce significantly more gesticulation when measured per 100 words than healthy controls; and

(2) Individuals with non-fluent aphasia will produce significantly more meaningladen gesture than both healthy controls and people with fluent aphasia.

The remaining investigations were exploratory rather than hypothesis driven.

\section{METHOD}

\section{Participants}

To investigate the gesture production patterns of people with aphasia, we analysed data from AphasiaBank, a multimedia database of discourse protocols gathered from persons with aphasia as well as healthy controls (http://www.talkbank.org/ AphasiaBank; MacWhinney, Fromm, Forbes, \& Holland, 2011). The database contains video samples of discourse in four genres (personal narrative, picture description, story re-telling, and procedural discourse) obtained using a standardised protocol. In addition, the results of the Western Aphasia Battery (WAB) Aphasia Quotient (Kertesz, 2007), short form Boston Naming Test (BNT) (Kaplan, Goodglass, \& Weintraub, 2001), Verb Naming Test (VNT) (Cho-Reyes \& Thompson, 2012), and the Aphasia Bank repetition test are included for almost all individuals with aphasia. Extensive demographic data is also reported, including gender, age, race, handedness, education, occupation, aphasia etiology and duration, aphasia type, site of lesion, mood, and history of communication disorders. The data were collected from different locations across the USA. 
The database available on September 2010 consisted of 102 participants with aphasia and 99 healthy control participants. In what follows they are referred to as the aphasia group and the control group, respectively. The participants selected for the current study are a subset of those we examined in a previous study (Sekine \& Rose, in press) that used the AphasiaBank samples to examine the gestures produced by people with aphasia during the Cinderella story re-tell task. In the previous study, 9 of the 102 individuals with aphasia were excluded from the analysis due to a WAB Aphasia Quotient above 93.8, the cut-off score for diagnosis of aphasia. An additional four individuals with aphasia were excluded from further analysis due to missing story re-tell samples, or poor camera views of their arms and hands that prevented gestures from being visualised clearly.

From the 89 participants in the aphasia group previously studied, 10 participants who produced gesture at least once were selected for the current study from each of the diagnostic categories of Broca's aphasia, Wernicke's aphasia, anomic aphasia, and conduction aphasia. Only six participants were selected from the transcortical motor aphasia group, as there were only six participants within this diagnostic category who produced gestures in the previous study. Participants with global aphasia were excluded from our analysis, as only two people in this diagnostic category were shown to produce gestures during the previous study. Thus, in the current study all 46 selected participants were individuals who produced gestures during the story retell task. In order to select the 10 participants from each diagnostic category we first selected 10 from the categories with low numbers of potential participants (Anomic, Wernicke), and then the 6 participants that were available in the Transcortical Motor category. We then chose the remaining participants so that they balanced the groups on severity, gender, age, and education level as far as possible.

In the previous study, 35 of 99 control participants were excluded from analysis due to missing story re-tell samples, or poor camera views of their arms and hands that prevented gestures from being analysed. Forty-seven of the remaining sixty-four control participants produced gestures at least once in their narratives. From these, 10 participants were selected by matching their age and educational levels with the aphasia group. The gender and age of the participants for each aphasia type and the control group are detailed in Table 1.

All participants were native English speakers or had English as their primary language (three were bilinguals who had acquired a second language after age six, three were early bilinguals, and two were multilingual), and resided in the USA. All individuals with aphasia had experienced a single, unilateral, left-hemisphere

TABLE 1

Gender, mean age, and standard deviation for aphasia group and control group

\begin{tabular}{lccc}
\hline & Male & Female & Mean age $(S D)$ \\
\hline Aphasia group & & & \\
Broca's & 7 & 3 & $56.6(13.3)$ \\
Wernicke's & 6 & 4 & $66.4(12.3)$ \\
Anomic & 5 & 5 & $58.7(13.0)$ \\
Conduction & 7 & 3 & $65.7(10.5)$ \\
TransMotor* & 3 & 3 & $68.8(12.5)$ \\
Control group & 5 & 5 & $57.0(15.9)$ \\
\hline
\end{tabular}

*TransMotor: Transcortical motor aphasia. 
stroke confirmed by neurological and ancillary examinations (Electroencephalography (EEG), Computed tomography (CT) scan) and were at least 6 months post-onset. Language profiles and aphasia severity ratings were completed according to the clinical criteria outlined by the WAB (Kertesz, 1982). Individual participant details are summarised in Appendix A.

\section{Discourse samples}

To examine the gestures that participants spontaneously produced in conversation, the videotaped personal narrative interview segment was extracted from the databank and analysed. The personal narratives for people with aphasia and controls consisted of responses to four questions (see Table 2). If the participant gave no response, after approximately 10 seconds the speech pathologist gave specific prompts (see the website for the detailed protocol). This proceeded until it was clear the participant could not continue. There was no time limit to answer the questions. The gestures and speech produced by people with aphasia and the controls during interviews were analysed.

\section{Data analysis}

In order to investigate the relationship between aphasia type and severity, and the type of gestures produced, gestures were coded into 12 types. Nine of the gesture codes had been used in previous studies (Cicone et al., 1979; Gullberg, 2006; McNeill, 1992) and three codes were created during our previous project (Sekine \& Rose, in press). Overall, the majority of the 12 gesture types coded in this study can be characterised as gesticulations ( 10 codes), with the addition of emblems and pantomimes (see Table 3). The classification of each gesture type in this study was mutually exclusive.

TABLE 2

Personal narrative prompt questions and extra prompt

People with aphasia

(1) "How do you think your speech is these days?"

If no response, ask "Are you having "trouble with your talking?"

(2) "Do you remember when you had your stroke? Please tell me about it."

\section{If no response, ask "Do you remember your} stroke?"

(3) "Tell me about your recovery. What kinds of things have you done to try to get better since your stroke?"

If no response, ask "Did you have any therapy after your stroke?"

(4) "Thinking back, can you tell me a story about something important that happened to you in your life? It could be happy or sad or from any time, from when you were a kid or more recently."
Controls

(1) "Could tell me what you remember about any illness or injury you've had?”

(2) "Tell me about your recovery from that illness (or injury). What kinds of things did you do to get better?"

(3) "Have you had any experience with people who have a difficult time communicating? Please tell me what the problems were and what you did about it."

(4) "Thinking back, can you tell me a story about something important that happened to you in your life? It could be happy or sad or from any time, from when you were a kid or more recently." 
TABLE 3

Type of gesture

\begin{tabular}{l} 
Type of gesture \\
\hline 1. Referential gestures \\
2. Concrete deictic \\
gestures \\
3. Pointing to self \\
4. Iconic observer \\
viewpoint gestures ${ }^{1}$ \\
(OVPT)
\end{tabular}

5. Iconic character viewpoint gestures (CVPT)

\section{Pantomime gestures}

7. Metaphoric gestures

8. Emblems

9. Time gestures

10. Beats

11. Letter gestures

12. Number gestures
Definition

Reference

Gullberg (2006)

Used to assign the entity of referents - such as objects, places, or characters in the story-into the space in front of a speaker where any concrete object is absent. The hand shape of the gesture usually takes the form of a pointing gesture or of holding some entity.

Indicate a concrete referent in the physical environment, such as a picture book or an item of actual clothing.

Gestures where the speaker points to their own body (mostly their chest) in order to refer to themselves.

Gestures that depict a concrete action, event or object as though the speaker is observing it. For example, to depict someone running, the speaker traces her index finger in the frontal space from left to right as if she is seeing the scene as an observer.

Gestures that use the speaker's own body in depicting a concrete action, event or object event, as though he himself is the character/object. For example, to depict someone running, he swings his arms back and forth, as if he is running.

Gestures that consist of two or more CVPT gestures that occur continuously within the same gesture unit. No matter how many CVPT gestures occur continuously, it is counted as a single pantomime. A gesture unit is defined as the period of time between successive rests of the limbs (McNeill, 1992).

Gestures that present an image of an abstract concept, such as knowledge or justice, language itself, the genre of the narrative, etc. It often has a cup-shaped hand shape.

Gestures whose form and meaning are established by the conventions of specific communities and can usually be understood without speech, such as thumb and pointer finger making a circle shape for "OK".

Indicate some space to denote a point in time, such as past (back of the body) or future (front of the body).

Movements that do not present a discernible meaning and are recognised by their prototypical repetitive movement characteristics timed with speech production.

Movements associated with writing letters in the air or on the desk/thigh with an empty hand or fingers.

Gestures that display numbers by using the speaker's fingers.
McNeill (1992)

Sekine \& Rose

(in press)

McNeill (1992)

McNeill (1992)

Sekine \& Rose

(in press)

McNeill (1992)

Kendon (1980)

Sekine \& Rose

(in press)

McNeill (1992)

Cicone et al. (1979)

Cicone et al. (1979)

\footnotetext{
${ }^{1}$ Iconic gestures are defined as gestures that depict some aspect of a concrete event or object by creating a homology to aspects of the event/object. They display, in their form and manner of execution, aspects of the same scene that speech also attempts to present.
} 
We used the program EUDICO Linguistic Annotator (ELAN) (http://www.lat-mpi. $\mathrm{eu} /$ tools/elan/) to annotate the gesture types produced in each sample.

General speech and gesture performances were analysed by calculating the total number of gestures, the frequency of gestures per 100 words, and the number of gestures per gesture unit. Word counts were undertaken using the method adopted by Lott (1999), whereby all lexical items that are part of the English language were counted, including neologistic paraphasias (e.g., "siglahb gabladubl" $=2$ words), phonemic paraphasias (e.g., "mermain" instead of "mermaid" = 1 word), initial phoneme repetitions (e.g., "As I s . . said" = 3 words), and self-corrected false starts (e.g., "In the sum . . . winter" $=4$ words; if the meaning of the false start word was obvious from the context (e.g., summer), it was counted as a word). Consistent with this method, filler and paralinguistic items were not counted. The items not counted included back channels (e.g., "mmm", "oh", "hmmm"), paralinguistic items, (e.g., "ehm", "ah", "oh"), or extra speech sounds (e.g., whistling, shushing, hissing).

A meaningful hand movement was only classified as a gesture when it had an identifiable beginning and a clear end. Coders had access to the speech signal while they were coding the gestures. However, gestures were coded even if there was no accompanying speech (for example, in the case of several pantomime productions). A gesture unit was defined as the period between successive rests of the limbs (Kendon, 1980). A gesture unit begins the moment the limb begins to move and ends when the limbs rest back to the original position (McNeill, 1992). A gesture unit often includes multiple gestures that consist of one or more movement phases.

\section{Reliability}

The first author coded the entire data set. To ensure the reliability of the speech and gesture coding, $60 \%$ of the data was re-analysed by three trained and independent native English speaking post-graduate students. Three participants from each aphasia sub-type (15 participants in total) and 6 participants from the control group were randomly selected and re-coded by the second coders according to the gesture type described above.

Point-to-point percentage agreement was calculated. The two coders agreed on the number of gestures $89 \%$ of the time for the aphasia group, and $90 \%$ of the time for the control group. The Cohen's kappa statistic was used to assess inter-rater reliability for gesture type. Agreement between the two independent coders for aphasia group (kappa $=.77$ ) and for the control group (kappa $=.85)$ was high. Any coding disagreements were resolved through discussion and subsequent consensus.

\section{RESULTS}

\section{Gesture and word frequency}

Descriptive statistics for gesture and word frequency are provided in Table 4. Discourse samples ranged from 124 to 825 seconds $(X=400.95, S D=176.17$ ). Descriptive statistics revealed that people with aphasia produced more gestures per 100 words than controls. To investigate the differences in gesture and word frequency between individuals from each aphasia type and the control group, we conducted ANOVA on the total number of gestures, words per second, gestures per 100 words, and gestures per gesture unit, with participant group as a between-subject factor (see 


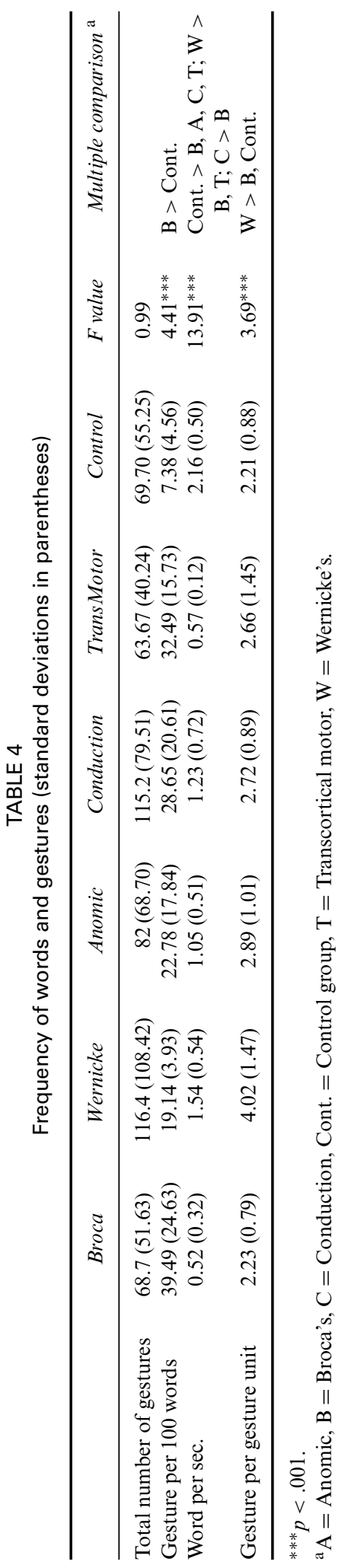


Table 4). A main effect of participant group was found for gestures per 100 words, $F(5,50)=4.41, p<.001, \eta^{2}=.31$, for words per second, $F(5,50)=13.91, p<.001$, $\eta^{2}=.58$, and for gestures per gesture-unit, $F(5,50)=3.69, p=.01, \eta^{2}=.27$.

Post-hoc tests (Tukey, $p<.05$ ) showed that the number of gestures per 100 words was significantly greater for people with Broca's aphasia than for controls. It also showed that the control group produced significantly more words per second than individuals with Broca's, anomic, conduction, and transcortical motor aphasia. The number of gestures per gesture unit was significantly greater for the Wernicke's aphasia group than for the Broca's aphasia and control groups.

\section{The frequency of gesture type per 100 words}

To examine the frequency of each gesture type while taking into consideration the speech fluency of various aphasia types, we calculated the gesture rate per 100 words. We then conducted ANOVA on each gesture type per 100 words with participant group as the between-subject factor (Table 5). A main effect of participant group was found for beats, $F(5,50)=3.28, p=.01, \eta^{2}=.25$, metaphoric gestures, $F(5,50)=2.45$, $p=.05, \eta^{2}=.20$, referential gestures, $F(5,50)=4.22, p=.003, \eta^{2}=.30$, emblems, $F(5,50)=6.73, p<.001, \eta^{2}=.40$, iconic character viewpoint gestures, $F(5,50)=4.31$, $p=.002, \eta^{2}=.43$, iconic observer viewpoint gestures, $F(5,50)=3.32, p=.01, \eta^{2}=.25$, and pantomime gestures, $F(5,50)=4.11, p=.003, \eta^{2}=.29$.

Post-hoc tests (Tukey, $p<.05$ ) revealed that the frequency of beats was significantly higher in the transcortical motor group than the conduction and control groups while the frequency of metaphoric gesture was significantly greater in the Wernicke's group than the conduction and control groups, and significantly greater in the anomic group than the control group. Further, the frequency of referential gesture was significantly greater in the conduction group than the Wernicke's and control groups. The frequency of emblems was significantly greater in the Broca's group than the Wernicke's, anomic, and control groups, and significantly greater in the transcortical motor than the control group. Similarly, the frequency of both iconic CVPT and pantomime gestures were significantly greater in the Broca's than the Wernicke's, anomic, transcortical motor aphasia, and control groups, and the frequency of iconic OVPT was significantly greater in the Broca's than Wernicke's and control groups.

\section{The relationship between gesture frequency and aphasia severity (WAB AQ) and fluency (WAB SSF)}

In order to examine the relationship between the frequency of gesture production and aphasia severity and fluency, Pearson's correlations were conducted by collapsing all aphasia types. As speech and language indices, we used the WAB Aphasia Quotient for severity and the Spontaneous Speech Fluency (WAB SSF) scores.

As shown in Table 6, the results revealed that the WAB AQ was significantly negatively correlated with concrete deictic $(r=-.39, p<.01)$, and positively correlated with referential gestures $(r=.46, p<.01)$. WAB SSF showed a significantly negative correlation with concrete deictics $(r=-.41, p<.01)$, emblems $(r=-.37, p<.05)$, iconic CVPT $(r=-.37, p<.05)$, iconic OVPT $(r=-.37, p<.05)$, and pantomimes $(r=-.37, p<.05)$. WAB SSF was positively correlated with the total number of words $(r=.44, p<.01)$ and gestures $(r=.35, p<.05)$ produced. 


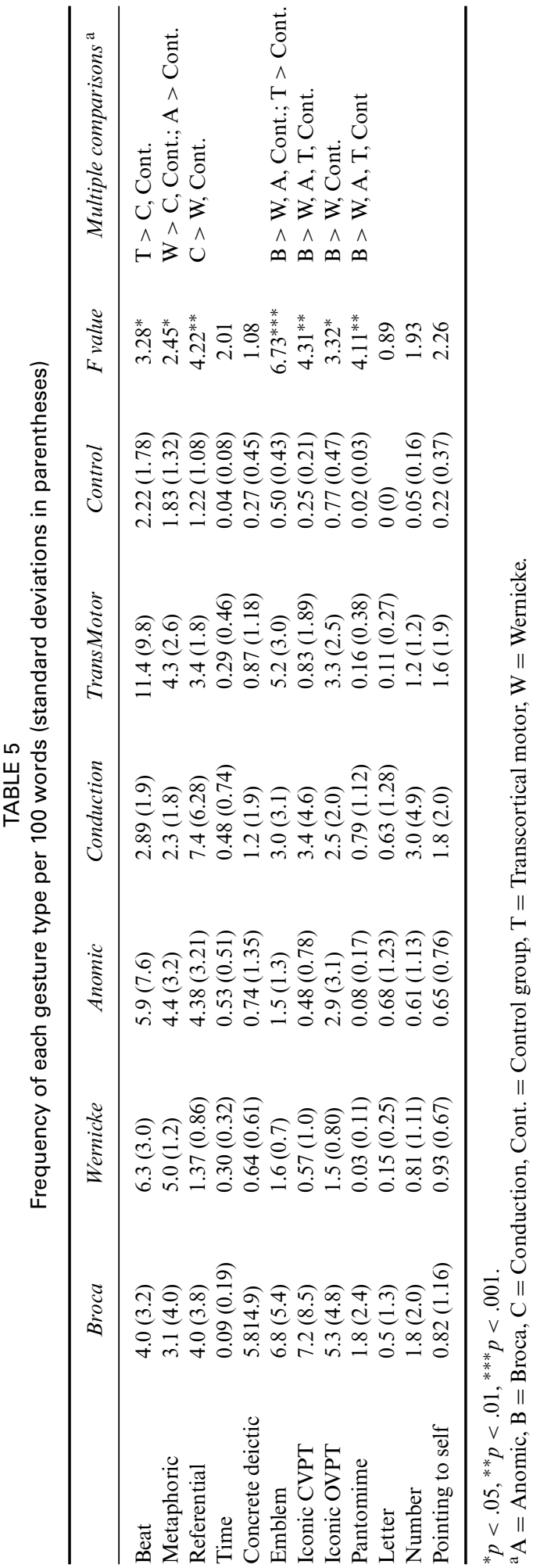


TABLE 6

Pearson's correlations between the frequency of gestures per 100 words and scores on WAB $\mathrm{AO}$ and SSF

\begin{tabular}{lcc}
\hline & $W A B A Q(N=44)$ & $W A B S S F(N=44)$ \\
\hline Beat & 0.17 & 0.20 \\
Concrete deictic & $-.377^{*}$ & $-.411^{* *}$ \\
Emblem & -0.09 & $-.372^{*}$ \\
Iconic CVPT & -0.29 & $-.445^{* *}$ \\
Iconic OVPT & -0.20 & $-.379^{*}$ \\
Letter & 0.10 & -0.03 \\
Metaphoric & -0.05 & 0.09 \\
Number & -0.11 & -0.28 \\
Point to self & 0.12 & -0.13 \\
Referential & $.335^{*}$ & 0.03 \\
Time & 0.07 & 0.03 \\
Pantomime & -0.28 & $-.430^{* *}$ \\
\hline
\end{tabular}

${ }^{*} p<.05,{ }^{* *} p<.01$.

\section{The influence of language condition on gesture production}

Finally, to examine if language factors such as fluency, aphasia severity, and naming ability predicted gesture production in people with aphasia, multiple linear regression analyses were used (Table 7). Stepwise inclusion of regressors was chosen as the method of analysis. To examine the influence of language condition on gesture production, we divided the gesture types into two groups. One group was called 'meaning-laden' gestures, consisting of the concrete deictic, emblem, iconic character viewpoint, iconic observer viewpoint, letter, number, and pointing to self gestures. Gestures in this group convey or indicate concrete meanings. The other group was designated "abstract gestures", consisting of the beat, metaphoric, referential, and time gestures. Gestures in this group either convey abstract meanings or do not convey any specific meaning.

First, we conducted multiple linear regression analysis with the dependent variable being the frequency of gesture per 100 words and the independent variables being the WAB SSF, WAB AQ, and BNT scores. The results revealed that WAB SSF $(\beta=.35$,

TABLE 7

Stepwise multiple linear regression analyses with the dependent variables, gesture frequency per 100 words and meaning-laden gesture per 100 words, and independent variables BNT, WAB $A Q$, and WAB SSF scores

\begin{tabular}{lll}
\hline & \multicolumn{2}{c}{ Regression coefficient } \\
\cline { 2 - 3 } $\begin{array}{l}\text { Independent } \\
\text { variables }\end{array}$ & Gesture frequency per 100 words & Meaning-laden gestures per 100 words \\
\hline BNT score & Excluded & $\begin{array}{l}\text { Excluded } \\
\beta=-.52^{\mathrm{b}} \\
\text { WAB SSF }\end{array}$ \\
WAB AQ & $\beta=.35^{\mathrm{a}}$ & Excluded \\
\hline
\end{tabular}

${ }^{\mathrm{a}} F(1,42)=5.71 ; p<.021, \mathrm{r}^{2}=.12$.

${ }^{\mathrm{b}} F(1,42)=15.32 ; p<.001, \mathrm{r}^{2}=.27$. 
$F(1,42)=5.71, p<.021)$ was a significant predictor. WAB AQ and BNT scores were not significant predictors $(\beta=.02$, n.s; $\beta=.16$, n.s. $)$. The overall model fit was $\mathrm{R}^{2}=.12$.

Next, we conducted multiple linear regression analysis with the dependent variable frequency of meaning-laden gestures per 100 words and the independent variables being WAB SSF, WAB AQ, and BNT scores. The results again indicated that WAB $\operatorname{SSF}(\beta=-.52, F(1,42)=15.32, p<.001)$ was a significant predictor. WAB AQ and BNT scores were not significant predictors $(\beta=-.09$, n.s.; $\beta=.07$, n.s. $)$. The overall model fit was $R^{2}=.27$. None of the language profiles were significant predictors for the frequency of abstract gestures per 100 words.

\section{DISCUSSION}

Our large-scale study enabled a more detailed examination of gesture patterns across five types of aphasia, for a range of aphasia severities, and with a more ecologically valid sampling technique than has previously been attempted. Further, we were able to use preliminary prediction analyses to investigate potential explanatory factors. Gesture production patterns in individuals with aphasia were found to differ from healthy controls in specific ways and these are discussed below.

Overall, individuals with Wernicke's aphasia produced a large number of gestures in their discourse. However, when speech fluency was taken into account and the amount of gestures per 100 words was analysed, individuals with Broca's, transcortical motor and conduction aphasia showed the highest rates of gesture production. Individuals with Broca's aphasia produced almost twice the amount of gestures per 100 words compared with those with Wernicke's aphasia. Further, the number of gestures produced within a gesture unit was significantly greater for those with Wernicke's aphasia. Yet, these individuals produced a low number of semantically rich emblem, iconic, and pantomime gestures and high number of beats and metaphoric gestures which are less communicatively meaningful. By contrast, the participants with Broca's and conduction aphasia produced high levels of meaning-laden gestures (concrete deictic, iconics, emblems, numbers, and pantomime gestures). These results are consistent with those found previously by Lott (1999), Carlomagno et al. (2005), and Cocks, Dipper, Pritchard, and Morgan (this issue). Furthermore, the findings are similar to those obtained in a previous study (Sekine \& Rose, in press) that used the same database but analysed single productions of gesture types during story re-tell samples. The previous study showed that more people with non-fluent aphasia (e.g., Broca's aphasia) produced meaning-laden gestures (e.g., deictic and iconic gestures) as compared to other types of aphasia ${ }^{1}$. This replication of findings indicates that the relationship between gesture types and aphasia types found in the current study seems robust.

\footnotetext{
${ }^{1}$ About $20 \%$ of meaning-laden gestures produced by people with Broca's aphasia were emblems. Some studies have argued that high use of emblems is linked to interview based discourse where there is a frequent use of closed ended questions requiring a simple Yes/No response (e.g., Cicone et al., 1997; Herrmann, Reichle, \& Lucius-Hoene, 1988). In this study, there were a total of 122 closed questions for the 46 participants with aphasia, and only 13 participants with aphasia responded to the closed questions with a total of 15 gestures. Of the 15 gesture responses, 7 were emblem, 4 were beats, 1 was a concrete deictic, 1 was a number, and 1 was a letter gesture. Given the total number of emblems (415) produced by all 46 participants during the interviews, 7 emblems represents $1.69 \%$ of the total number of emblems. Thus, in our study, the vast majority of emblems were produced during spontaneous speech rather than in response to closed questions.
} 
The pattern of meaning-laden gesture production found in the current study seems consistent with two main ideas that we will term the Dual-Factor hypothesis: Factor A) whether the speaker perceives a need to gesture for meaning transfer and Factor B) the actual capacity of the speaker to represent meaning/propositions in gesture. Considering the need to gesture for communication, clearly individuals with a limited ability to represent thought in language due to their aphasic impairment are likely candidates for gesture-based communication. Such ideas are consistent with de Ruiter's Mutually Adaptive Modalities assumption from the Sketch model of speech and gesture production (see de Ruiter, 2000). Individuals with Broca's and Wernicke's aphasia both have difficulties in representing thought in language and therefore might have a high need to utilise gesture-based communication (Factor A). However, their capacity to represent thought in gesture (Factor B) might set them apart. Recently, Hogrefe et al. (2012) found a strong relationship between the integrity of semantic processing and gesture diversity in individuals with severe aphasia, and Cocks and colleagues (this issue) found strong correlations between semantic processing and gesture production during word retrieval failure. Although AphasiaBank does not provide direct measures of semantic processing, it is likely that the participants with Broca's, transcortical motor and conduction aphasia studied here had better semantic processing than those with Wernicke's aphasia (Hagoort, 1993).

The Dual-Factor hypothesis for understanding aphasic meaning-laden gesture production patterns found partial support in the results of the fluency analyses here, such that fluency was shown to be a stronger predictor of gesture production patterns than aphasia severity. While the production of concrete deictic and referential gesture was significantly negatively correlated with aphasia severity, there were stronger negative relationships between fluency and concrete deictic, emblem, iconic, and pantomime gesture production, and a positive correlation between the total number of gestures produced and fluency scores. Further, the regression analyses confirmed that fluency was a better predictor of gesture production, and more specifically production of meaning-laden gestures, than aphasia severity or naming ability. However, only $27 \%$ of the variance was accounted for by the fluency factor. This is not surprising when considering that several aphasia types might have similarly high levels of fluency (e.g., Wernicke's; anomic) but very different needs to represent meaning in gesture due to their different linguistic abilities (Wernicke's high need; anomic low need).

While the integrity of semantic processing is a likely candidate to explain some of the "capacity to represent meaning in gesture" factor (Factor B), other possible candidates include mental flexibility (capacity to shift mental set), capacity to recognise error and generate solutions (Purdy \& Koch, 2006), and non-verbal reasoning skills (Lambon Ralph, Snell, Fillingham, Conroy, \& Sage, 2010). These possible predictors of gesture production patterns require further empirical investigation.

This study has provided firm evidence supporting the previously discussed idea that people with Broca's, conduction and transcortical motor aphasia are capable of producing high numbers of meaning-laden iconic gestures. Given the need for alternative communication strategies in these populations (either as a full lexical substitute or as co-verbal support), gesture should be routinely considered by speech-language pathologists (SLPs) planning intervention strategies (see Rose, 2006 for a review of the issues; and Rose et al., this issue for systematic review of gesture treatment studies). Sampling discourse is an obvious assessment strategy for a number of reasons but paying attention to gesture production patterns during such assessment needs to be considered. A study by Sekine \& Rose (in press), which analysed the gestures produced by people 
with aphasia in the story re-telling task, found a similar relationship between gesture type and aphasia type as the current study. Thus, our results suggest that sampling story re-tell or conversation may produce similar gesture production patterns.

People with anomic aphasia also produced the full range of gestures during discourse. Although their rate of use of pantomime, emblem, and iconic gestures did not match that of other aphasia types, such capacity may have utility during their frequent word retrieval failures, and this too requires further study as a possible therapeutic device. While people with Wernicke's aphasia in this study had lower production of iconic gesture forms in discourse than other types of aphasia, they did show some capacity to produce these gestures. Whether this capacity can be harnessed therapeutically requires further examination (see Carlomagno, Zulian, Razzano, De Mercurio, \& Martini (2012) for a recent pilot study on this topic).

One limitation of utilising the AphasiaBank samples in this study was the lack of detail available concerning semantic- and other cognitive-processing skills. Similarly, there is little or no detail about limb apraxia in AphasiaBank, and Hogrefe et al. (2012) have demonstrated a possible impact of limb apraxia on gesture comprehensibility in individuals with severe aphasia. A further limitation of the current study was the omission of people with global aphasia. Only two individuals with global aphasia were available from the AphasiaBank samples and thus we were required to remove these from statistical analyses. Further, we only had two individuals in the study with very severe aphasia (WAB AQ $<25$ ). Future studies should aim to include the full range of aphasia types and severities.

We believe it is now timely to conduct a large and comprehensive study of gesture patterns in aphasic discourse, examining the predictors discussed here and including a full range of praxis and cognitive measures. Our study has helped to clarify several factors that require further examination in such a study. Another pressing question is the communicative value of the gestures produced. While the meaning-laden gestures we documented here are arguably communicative, empirical work is required to verify their communicative value and thus support therapeutic goals targeting their use.

\section{REFERENCES}

Ahlsén, E. (1991). Body communication as compensation for speech in a Wernicke's aphasia —a longitudinal study. Journal of Communication Disorders, 24, 1-12.

Beattie, G., \& Coughlan, J. (1998). Do iconic gestures have a functional role in lexical access? An experimental study of the effects of repeating a verbal message on gesture production. Semiotica, 119, 221-249.

Behrmann, M., \& Penn, C. (1984). Non-verbal communication in aphasia. British Journal of Disorders of Communication, 19, 155-176.

Carlomagno, S., Pandolfi, M., Martini, A., Di Iasi, G., \& Cristilli, C. (2005). Coverbal gestures in Alzheimer's type dementia. Cortex, 41, 535-546.

Carlomagno, S., Zulian, N., Razzano, C., De Mercurio, I., \& Martini, A. (2012). Coverbal gestures in the recovery from severe fluent aphasia: A pilot study. Journal of Communication Disorders, 46, 84-99.

Cho-Reyes, S., \& Thompson, C. K. (2012). Verb and sentence production and comprehension: Northwestern assessment of Verbs and Sentences (NAVS). Aphasiology, 6, 1250-1277.

Cicone, M., Wapner, W., Foldi, N., Zurif, E., \& Gardner, H. (1979). The relation between gesture and language in aphasic communication. Brain and Language, 8, 324-349.

Cocks, N., Dipper, L., Pritchard, M., \& Morgan, G. (this issue). The impact of impaired semantic knowledge on spontaneous iconic gesture production. Aphasiology. 
Cruice, M., Worrall, L., Hickson, L., \& Murison, R. (2005). Measuring quality of life: Comparing family members' and friends' ratings with those of their aphasic partners. Aphasiology, 19, 111-129.

Daniloff, J., Fritelli, G., Buckingham, H., Hoffman, P., \& Daniloff, R. (1986). Amer-Ind versus ASL: Recognition and imitation in aphasic subjects. Brain and Language, 28, 95-113.

de Ruiter, J. (2000). The production of gesture and speech. In D. McNeill (Ed.), Language and Gesture (pp. 284-311). Cambridge: Cambridge University Press.

Dickey, L., Kagan, A., Lindsay, P., Fang, J., Rowland, A., \& Black, S. (2010). Incidence and profile of inpatient stroke induced aphasia in Ontario, Canada. Archives of Physical Medicine and Rehabilitation, 91, 196-202.

Duffy, J., Duffy, R., \& Mercaitis, A. (1984). Comparison of the performances of a fluent and a nonfluent aphasic on a pantomimic referential task. Brain and Language, 21, 260-273.

Efron, D. (1972). Gesture, race, and culture. The Hague: Mouton.

Ekman, P., \& Friesen, W.V. (1969). The repertoire of nonverbal behavior: Categories, origins, usage, and coding. Semiotica, 1, 49-98.

Engelter, S. T., Gostynski, M., Papa, S., Frei, M., Born, C., Ajdacic-Gross, V., .. . Lyrer, P. A. (2006). Epidemiology of aphasia attributable to first ischemic stroke: Incidence, severity, fluency, etiology, and thrombolysis. Stroke, 37, 1379-1384.

Frick-Horbury, D., \& Guttentag, R. (1998). The effects of restricting hand gesture production on lexical retrieval and free recall. American Journal of Psychology, 3, 43-62.

Glosser, G., Wiener M., \& Kaplan, E. (1986). Communicative gestures in aphasia. Brain and Language, 27, 345-359.

Goldin-Meadow, S., McNeill, D., \& Singleton, J. (1996). Silence is liberating: Removing the handcuffs on grammatical expression in the manual modality. The Psychological Review, 103, 34-55.

Goodwin, C. (2000). Gesture, aphasia, and interaction. In D. McNeill (Ed.), Language and gesture (pp. 84-98). Cambridge, UK: Cambridge University Press.

Gullberg, M. (2006). Handling discourse: Gestures, reference, tracking, and communication strategies in early L2. Language Learning, 56, 155-196.

Haberman, S. J. (1973). The analysis of residuals in cross-classified tables. Biometrics, 29, 205-220.

Hagoort, P. (1993). Impairment of lexical-semantic processing in aphasia: Evidence from the processing of lexical ambiguities. Brain and Language, 45, 189-232.

Herrmann, M., Reichle, T., \& Lucius-Hoene, G. (1988). Nonverbal communication as a compensation strategy for severely nonfluent aphasics? A quantitative approach. Brain and Language, 33, 41-54.

Hilari, K., Northcott, S., Roy, P., Marshall, J., Wiggins, R., Chataway, J., \& Ames, D. (2010). Psychological distress after stroke and aphasia: The first six months. Clinical Rehabilitation, 24, 181-190.

Hogrefe, K., Zeigler, W., Weidinger, N., \& Goldenberg, G. (2012). Non-verbal communication in severe aphasia: Influence of aphasia, apraxia or semantic processing. Cortex, 48, 952-962.

Kadish, J. (1978). A neuropsychological approach to the study of gesture and pantomime in aphasia. South African Journal of Communication Disorders, 25, 102-117.

Kaplan, E., Goodglass, H., \& Weintraub, S. (2001). Boston naming test (2nd ed). Austin, TX: Pro-Ed.

Kendon, A. (1980). Gesticulation and speech: Two aspects of the process of utterance. In M. Key (Ed.), The relationship of verbal and nonverbal communication (pp. 207-227). The Hague: Mouton.

Kertesz, A. (2007). Western aphasia battery. San Antonio, TX: PsychCorp.

Kita, S., \& Davies, T. (2009). Competing conceptual representations trigger co-speech representational gestures. Language and Cognitive Processes, 24, 761-775.

Kita, S., \& Ozyurek, A. (2003). What does cross-linguistic variation in semantic coordination of speech and gesture reveal? Evidence for an interface representation of spatial thinking and speaking. Journal of Memory and Language, 48, 16-32.

Lambon Ralph, M., Snell, C., Fillingham, J., Conroy, P., \& Sage, K. (2010). Predicting the outcome of anomia therapy for people with aphasia post CVA: Both language and cognitive status are key predictors. Neuropsychological Rehabilitation, 20, 289-305.

Lanyon, L., \& Rose, M. (2009). Do the hands have it? The facilitation effects of arm and hand gesture on word retrieval in aphasia. Aphasiology, 23, 809-822.

Le May, A., David, R., \& Thomas, A. (1988). The use of spontaneous gesture by aphasic patients. Aphasiology, 2, 137-145.

Lott, P. (1999). Gesture and aphasia. Berlin: Peter Lang.

MacWhinney, B., Fromm, D., Forbes, M., \& Holland, A. (2011). AphasiaBank: Methods for studying discourse. Aphasiology, 25, 1286-1307. 
McNeill, D., (1992). Hand and mind: What gestures reveal about thought. Chicago, IL: University of Chicago Press.

McNeill, D. (Ed.) (2000). Introduction. Language and gesture (pp. 1-10). Cambridge: Cambridge University Press.

McNeill, D. (2005). Gesture and thought. Chicago, IL: University of Chicago Press.

Melinger, A., \& Levelt, W. (2004). Gesture and the communication intention of the speaker. Gesture, 4, 119-141.

Morrell-Samuels, P., \& Krauss, R. (1992). Word familiarity predicts temporal asynchrony of hand gestures and speech. Journal of Experimental Psychology, Learning, Memory and Cognition, 18, 615-622.

Pedelty, L. L. (1987). Gesture in aphasia. Chicago, IL: Department of Behavioral Sciences, University of Chicago.

Pickett, L. (1974). Assessment of gesture and pantomimic deficit in aphasic patients. Acta Symbolica, 5, 69-86.

Purdy, M., \& Koch, A. (2006). Prediction of strategy usage by adults with aphasia. Aphasiology, 20, $337-348$.

Pyers, J., Grossmith, S., Magid, R., Dikanovic, M., Gollan, T., \& Emmorey, K. (2010, May). Individual differences in the role of gesture in lexical retrieval. Poster presented at the 22 Annual Meeting of the Association for Psychological Science, Boston, MA.

Rauscher, F., Krauss, R., \& Chen, Y. (1996). Gesture, speech and lexical access: The role of lexical movements in speech production. Psychological Science, 7, 226-230.

Rose, M. (2006). The utility of gesture treatments in aphasia. International Journal of Speech Language Pathology, 8, 92-109.

Rose, M., \& Douglas, J. (2001). The differential facilitatory effects of gesture and visualisation processes on object naming in aphasia. Aphasiology, 15, 977-990.

Rose, M., \& Douglas, J. (2003). Limb apraxia, pantomime, and lexical gesture in aphasic speakers: Preliminary findings. Aphasiology, 17, 453-464.

Rose, M., Raymer, A., Lanyon, L., \& Attard, M. C. (this issue). A systematic review of gesture treatments for post-stroke aphasia. Aphasiology,

Sekine, K., \& Rose, M. (in press). The relationship between aphasia type and gesture production in people with aphasia.

Wang, L., \& Goodglass, H. (1992). Pantomime, praxis, and aphasia. Brain and Language, 42, 402-418. 
APPENDIX A

Profile of participants in the aphasia group and the control group: gender, age, handedness, aphasia type, aphasia severity, apraxia of speech, hemiplegia, race, and years of education

\begin{tabular}{|c|c|c|c|c|c|c|c|c|c|}
\hline Participant & Gender & Age & $\begin{array}{c}\text { Handed- } \\
\text { ness }\end{array}$ & Aphasia type & $\begin{array}{c}\text { Aphasia } \\
\text { severity } \\
(W A B \\
A Q)^{1}\end{array}$ & $\begin{array}{c}\text { Apraxia } \\
\text { of } \\
\text { speech }^{2}\end{array}$ & $\begin{array}{c}\text { Hemipl- } \\
\text { egia }^{3}\end{array}$ & Race $^{4}$ & $\begin{array}{l}\text { Years of } \\
\text { education }\end{array}$ \\
\hline adler09a & Female & 41.7 & A & Anomic & 92.8 & $\mathrm{Y}$ & RW & WH & 16 \\
\hline adler21a & Male & 36.0 & Right & Anomic & 88 & $\mathrm{Y}$ & LW & WH & 16 \\
\hline elman07a & Male & 65.5 & Right & Anomic & 63.4 & $\mathrm{~N}$ & $\mathrm{RP}$ & WH & 16 \\
\hline scale02a & Male & 57.5 & A & Anomic & 70.6 & $\mathrm{Y}$ & $\mathrm{RP}$ & WH & 22 \\
\hline scale $08 \mathrm{a}$ & Male & 72.8 & Right & Anomic & 87.9 & $\mathrm{~N}$ & NM & WH & 12 \\
\hline scale17a & Female & 54.2 & A & Anomic & 91.8 & $\mathrm{~N}$ & $\mathrm{RP}$ & AA & 16 \\
\hline wright $202 a$ & Female & 63.2 & Right & Anomic & 89.5 & $\mathrm{~N}$ & RW & WH & 14 \\
\hline kansas12a & Male & $\mathrm{U}$ & $\mathrm{U}$ & Anomic & 68.7 & $\mathrm{~N}$ & $\mathrm{RP}$ & $\mathrm{U}$ & $\mathrm{U}$ \\
\hline tucson03a & Female & 46.8 & Right & Anomic & 67.6 & $\mathrm{~N}$ & NM & WH & 19 \\
\hline thompson04a & Female & 79.6 & Right & Anomic & 72.4 & $\mathrm{U}$ & NM & WH & 16 \\
\hline $\mathrm{cmu} 02 \mathrm{a}$ & Male & 35.9 & Right & Broca & $\mathrm{U}$ & $\mathrm{Y}$ & $\mathrm{RP}$ & WH & 16 \\
\hline adler11a & Male & 80.9 & Right & Broca & 17 & $\mathrm{Y}$ & RW & WH & 16 \\
\hline adler13a & Male & 52.4 & Left & Broca & 55.8 & $\mathrm{Y}$ & RW & WH & 18 \\
\hline adler25a & Male & 66.2 & Right & Broca & 77.6 & $\mathrm{Y}$ & $\mathrm{RP}$ & WH & 16 \\
\hline kempler04a & Female & 60.3 & Right & Broca & 54.6 & $\mathrm{~N}$ & $\mathrm{RP}$ & WH & 16 \\
\hline tucson $13 \mathrm{a}$ & Male & 68.2 & Right & Broca & $\mathrm{U}$ & $\mathrm{U}$ & RW & WH & 13 \\
\hline tucson $14 \mathrm{a}$ & Female & 53.9 & Right & Broca & 48.9 & $\mathrm{U}$ & RW & MI & 12 \\
\hline elman03a & Male & 55.2 & Right & Broca & 66.2 & $\mathrm{Y}$ & $\mathrm{RP}$ & AS & $20+$ \\
\hline wright $201 \mathrm{a}$ & Male & 55.2 & Right & Broca & 57.6 & $\mathrm{Y}$ & RW & WH & 14 \\
\hline wright $206 a$ & Female & 39.0 & Right & Broca & 53.7 & $\mathrm{~N}$ & RW & WH & 14 \\
\hline adler02a & Male & 69.8 & Right & Conduction & 74.9 & $\mathrm{Y}$ & RW & WH & 20 \\
\hline adler14a & Male & 71.4 & A & Conduction & 83 & $\mathrm{~N}$ & RW & AA & 13 \\
\hline tucson08a & Female & 56.6 & Right & Conduction & 73.4 & $\mathrm{Y}$ & RW & WH & 13 \\
\hline tucson $12 \mathrm{a}$ & Male & 72.7 & Right & Conduction & 24 & $\mathrm{U}$ & NM & WH & 16 \\
\hline thompson01a & Male & 44.6 & Right & Conduction & 93 & $\mathrm{~N}$ & NM & WH & 17 \\
\hline elman02a & Female & 81.9 & Right & Conduction & 61.7 & $\mathrm{Y}$ & NM & WH & 18 \\
\hline scale04a & Female & 61.8 & Right & Conduction & 72.8 & $\mathrm{Y}$ & RW & WH & 12 \\
\hline scale13a & Male & 73.2 & Right & Conduction & 70.1 & $\mathrm{Y}$ & RW & WH & 16 \\
\hline scale15a & Male & 58.3 & Left & Conduction & 68.3 & $\mathrm{~N}$ & $\mathrm{RP}$ & WH & 12 \\
\hline wright $203 \mathrm{a}$ & Male & 66.4 & Right & Conduction & 76.3 & $\mathrm{~N}$ & NM & WH & 18 \\
\hline thompson $02 \mathrm{a}$ & Female & 47.2 & Right & TransMotor & 86.5 & $\mathrm{Y}$ & $\mathrm{RP}$ & WH & 16 \\
\hline scale $05 \mathrm{a}$ & Male & 63.7 & Right & TransMotor & 73.2 & $\mathrm{Y}$ & $\mathrm{RP}$ & AA & 13 \\
\hline adler18a & Male & 71.5 & Right & Transmotor & 59.8 & $\mathrm{~N}$ & $\mathrm{RP}$ & WH & 12 \\
\hline thompson09a & Female & 74.0 & Right & TransMotor & 79.3 & $\mathrm{U}$ & NM & WH & 14 \\
\hline scale19a & Male & 83.6 & Right & TransMotor & 67.8 & $\mathrm{~N}$ & $\mathrm{RP}$ & WH & 15 \\
\hline adler04a & Female & 75.5 & Right & TransMotor & 72.6 & $\mathrm{Y}$ & LW & WH & 20 \\
\hline kansas14a & Female & 77.4 & Right & Wernicke & 46.2 & $\mathrm{~N}$ & RW & WH & 17 \\
\hline TAP08a & Female & 54.6 & Right & Wernicke & 34.5 & $\mathrm{~N}$ & NM & WH & 16 \\
\hline scale14a & Male & 63.7 & Right & Wernicke & 43.1 & $\mathrm{~N}$ & $\mathrm{RP}$ & WH & 20 \\
\hline tucson $15 \mathrm{a}$ & Male & 74.1 & Right & Wernicke & 67.4 & $\mathrm{~N}$ & $\mathrm{U}$ & WH & 15 \\
\hline adler23a & Male & 81.3 & Right & Wernicke & 46.8 & $\mathrm{~N}$ & NM & WH & $\mathrm{U}$ \\
\hline elman12a & Male & 57.4 & Right & Wernicke & 74.4 & $\mathrm{~N}$ & NM & WH & $20+$ \\
\hline elman14a & Female & 76.3 & Right & Wernicke & 65.7 & $\mathrm{~N}$ & NM & AA & 17 \\
\hline adler06a & Male & 70.6 & Right & Wernicke & 28.2 & $\mathrm{~N}$ & NM & WH & 12 \\
\hline kansas05a & Female & 69.9 & Right & Wernicke & 33.4 & $\mathrm{~N}$ & NM & WH & 18 \\
\hline scale $24 a$ & Male & 61.8 & Right & Wernicke & 40.2 & $\mathrm{~N}$ & RW & WH & 18 \\
\hline
\end{tabular}


APPENDIX A

(Continued)

\begin{tabular}{|c|c|c|c|c|c|c|c|c|c|}
\hline Participant & Gender & Age & $\begin{array}{l}\text { Handed- } \\
\text { ness }\end{array}$ & Aphasia type & $\begin{array}{c}\text { Aphasia } \\
\text { severity } \\
(W A B \\
A Q)^{1}\end{array}$ & $\begin{array}{c}\text { Apraxia } \\
\text { of } \\
\text { speech }^{2}\end{array}$ & $\begin{array}{c}\text { Hemipl- } \\
\text { egia }^{3}\end{array}$ & Race $^{4}$ & $\begin{array}{c}\text { Years of } \\
\text { education }\end{array}$ \\
\hline capilouto25a & Female & 71.4 & Right & Control & NA & NA & NA & WH & 15 \\
\hline wright02a & Female & 73.4 & Right & Control & NA & NA & NA & WH & 16 \\
\hline wright08a & Female & 51.0 & Right & Control & NA & NA & NA & WH & 12 \\
\hline wright $21 \mathrm{a}$ & Female & 36.0 & Right & Control & NA & NA & NA & WH & 16 \\
\hline wright $38 \mathrm{a}$ & Male & 47.5 & Right & Control & NA & NA & NA & WH & 12 \\
\hline wright $45 \mathrm{a}$ & Male & 76.5 & Right & Control & NA & NA & NA & WH & 14 \\
\hline wright $46 a$ & Male & 34.4 & Right & Control & NA & NA & NA & WH & 12 \\
\hline wright $49 a$ & Male & 48.7 & Right & Control & NA & NA & NA & WH & 20 \\
\hline wright $52 \mathrm{a}$ & Male & 64.1 & Right & Control & NA & NA & NA & WH & 20 \\
\hline wright59a & Female & 70.6 & Right & Control & NA & NA & NA & WH & 16 \\
\hline
\end{tabular}

${ }^{1} \mathrm{U}$ : unavailable.

${ }^{2} \mathrm{Y}$ : yes, N: no, U: unavailable.

${ }^{3}$ RP: right sided hemiplegia, LP: left sided hemiplegia, RW: right sided hemiparesis, LW: left sided hemiparesis, NM: no motor problems, U: unavailable.

${ }^{4}$ WH: white, AA: African American, AS: Asian, MI: mixed, U: unavailable. 